\title{
Subarachnoid haemorrhage in the elderly: a necropsy study of the association with cerebral amyloid angiopathy
}

\author{
Masahito Yamada, Yoshinori Itoh, Eiichi Otomo, Michio Hayakawa, Tadashi Miyatake
}

\begin{abstract}
To clarify the contribution of cerebral amyloid angiopathy (CAA) to subarachnoid haemorrhage (SAH) in the elderly, relationships between SAH and CAA were investigated in 997 necropsy cases aged 60 years or older. Primary SAH (bleeding from subarachnoid vessels) was found in 15 cases $(1 \cdot 5 \%)$. There was no case in which primary SAH was clearly attributed to CAA. Secondary SAH [secondary rupture of intracerebral haemorrhage (ICH) through the cortex to the subarachnoid space] was found in 23 patients $(2 \cdot 3 \%)$. In $11(48 \%)$ of them, ICH with secondary SAH was associated with CAA. The results indicated that primary SAH is rarely related to CAA, however, CAA is the most frequent cause of ICH accompanying secondary SAH in the elderly.
\end{abstract}

(F Neurol Neurosurg Psychiatry 1993;56:543-547)

Subarachnoid haemorrhage (SAH) occurs when subarachnoid blood vessels are primary sources of bleeding ("primary SAH"), or when ICH secondarily ruptures through the cortex into the subarachnoid space ("secondary SAH"). ${ }^{1}$ About $13-22 \%$ of primary SAH arise without any known aetiology despite intensive investigations. ${ }^{2}$

Cerebral amyloid angiopathy (CAA) is cerebrovascular amyloid deposition found in the brains of the elderly and patients with dementia of Alzheimer type (DAT), and other disorders including hereditary cerebral haemorrhage with amyloidosis. ${ }^{3-10} \mathrm{CAA}$ is one of the causes of cerebrovascular disease (CVD) in elderly individuals and demented patients, including intracerebral haemorrhage (ICH) of subcortical or lobar type, multiple cortical cerebral infarctions, and leukoencephalopathy. ${ }^{58-23}$

It is common in brains with CAA that subarachnoid leptomeningeal and cortical blood vessels are heavily infiltrated with amyloid. ${ }^{3-23}$ Meningeal haemosiderosis has been described in brains with CAA. ${ }^{9}$ Furthermore, CAArelated haemorrhage often occurs with meningeal signs, which indicates an association with SAH. ${ }^{151618} 19$

In this report we investigated relationships between SAH and CAA to clarify the contribution of CAA to SAH in elderly individuals.
Primary SAH is rarely related to CAA, but CAA is the most frequent cause of secondary SAH developing from ICH in the elderly.

\section{Subjects and methods}

From 1000 consecutive necropsy cases in a large geriatric hospital, we investigated 997 cases aged 60 years or older $(\mathrm{M} / \mathrm{F}=$ $369 / 628$ ). Their ages ranged from 60 to 102 years with an average of 83 years. The geriatric hospital was established as an annex to old people's homes in which about 1000 people live. Most of the patients admitted to the hospital were from the homes. Approximately $30-40 \%$ of patients admitted to the hospital had dementia. These 997 patients were clinicopathologically investigated for SAH and ICH. In most of the patients, intracranial haemorrhages developed in the homes or in hospital. The brains with SAH or ICH were analysed for the presence of CAA. Formalinfixed, paraffin-embedded sections of cerebrum and cerebellum as shown in a previous study $^{8}$ were stained with haematoxylin and eosin, Congo red, Klüver-Barrera, Bodian, and Elastica-Masson stain. Congo red-positive material which shows green birefringence with polarised light was identified as amyloid. Degrees of CAA were classified as mild (affected in 1-3 of 10 vessels), moderate (4-7 of 10 vessels), and marked (8-10 of 10 vessels). To characterise the amyloid protein of CAA, brain sections from patients with CAA were stained with a polyclonal antibody to amyloid beta/A4 protein using immunoperoxidase procedures as previously described. ${ }^{24}$ Vascular changes were carefully observed in brains involved with CAA. When CAA of moderate or marked degree was identified at the bleeding site without any obvious cause of haemorrhage, the haemorrhage was considered to be associated with CAA.

\section{Results}

PRIMARY SAH AND CAA

Primary SAH was found in $15(1.5 \%)$ (ages; 62-96 years with an average of 83 years, $M / F$ $=1 / 14$ ) of the 997 patients. Among the 15 patients with primary SAH, one patient had DAT and three had dementia of the vascular type, as diagnosed by clinical and neuropathological findings. The aetiology of primary SAH in these 15 patients is shown in table 1. Primary SAH was most frequently associated with generalised bleeding disorders (40\%). All patients with bleeding disorders 
Table 1 Aetiology of primary subarachnoid haemorrhage.

\begin{tabular}{ll}
\hline Aetiology & Number of patients (\%) \\
\hline Generalised bleeding disorders & $6(40 \%)$ \\
Cerebral arterial aneurysm & $5(33 \%)$ \\
Trauma & $1(7 \%)$ \\
Unknown & $3(20 \%)$ \\
Total & 15 \\
\hline
\end{tabular}

were suffering from disseminated intravascular coagulation (DIC).

Brain sections of these patients with primary SAH were pathologically investigated for the presence of CAA. In one of 6 patients with a generalised bleeding disorder, CAA of moderate degree was observed. This patient, with DIC, also showed bleeding in other organs including the gastrointestinal tract, and the DIC was considered to be the major

Table 2 Incidence of secondary subarachnoid haemorrhage (SAH) in patients with intracerebral haemorrhage (ICH)

\begin{tabular}{lcc}
\hline & \multicolumn{2}{l}{ Incidence of secondary $S A H$} \\
\cline { 2 - 3 } Location of ICH & [Number of patients](\%) & [Number of haematomas](\%) \\
\hline Cerebral hemispheres & $17 / 29(59 \%)$ & $20 / 33(61 \%)$ \\
Cerebellum & $5 / 14(36 \%)$ & $5 / 16(31 \%)$ \\
Brainstem & $1 / 5(20 \%)$ & $1 / 5(20 \%)$ \\
Basal ganglia or thalamus & $0 / 66(0 \%)$ & $0 / 71(0 \%)$ \\
Total & $23 / 101(23 \%)$ & $26 / 125(21 \%)$ \\
\hline
\end{tabular}

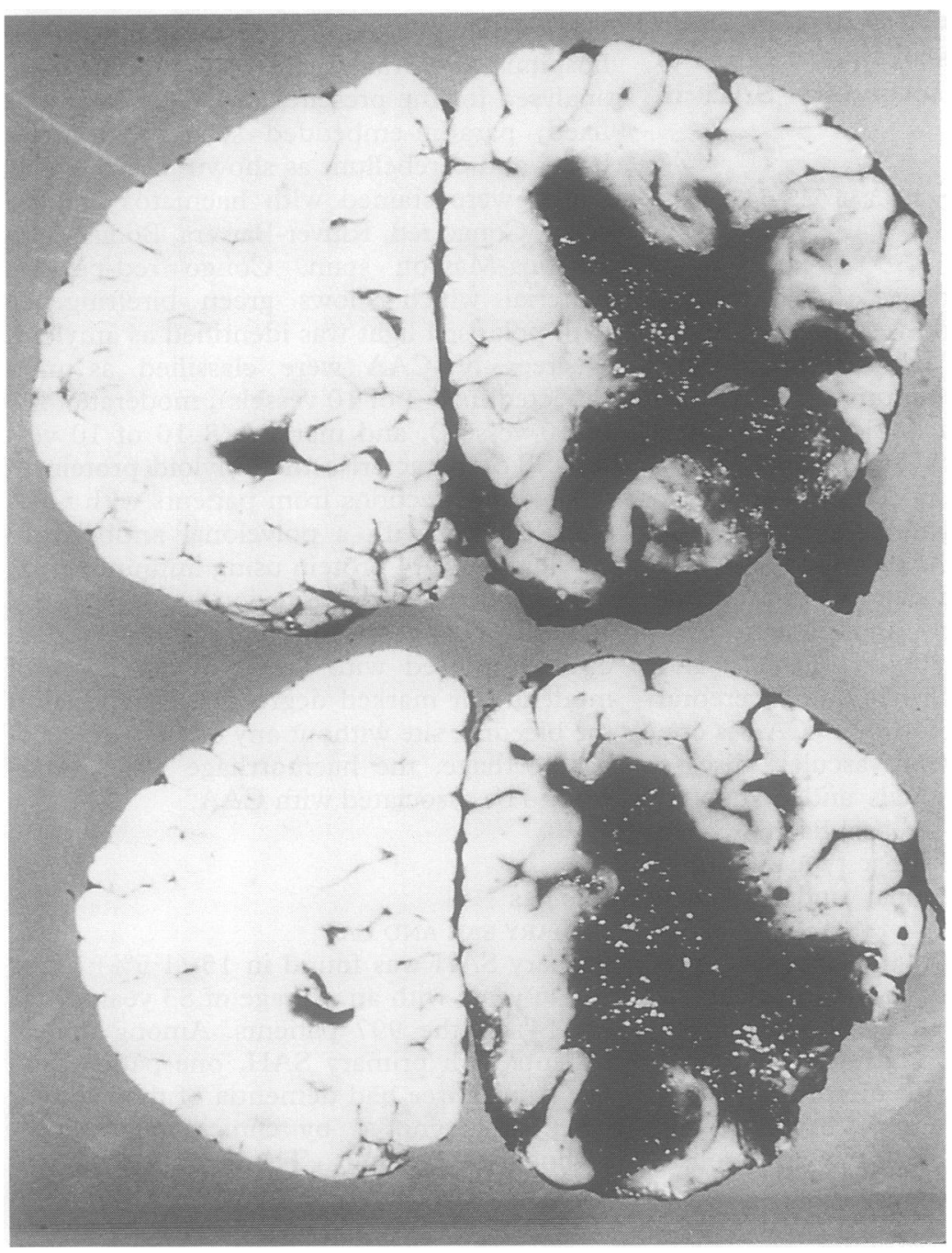

Figure 1 An intracerebral haematoma with secondary subarachnoid haemorrhage in the left occipital lobe in an 82 year old patient with severe cerebral amyloid angiopathy.
Table 3 Aetiology of intracerebral haemorrhages with secondary subarachnoid haemorrhage

\begin{tabular}{lcl}
\hline Aetiology & $\begin{array}{l}\text { Number of } \\
\text { patients (\%) }\end{array}$ & $\begin{array}{l}\text { Number of } \\
\text { haematomas (\%) }\end{array}$ \\
\hline CAA & $11(48 \%)$ & $12(46 \%)$ \\
Hypertension & $7(30 \%)$ & $8(31 \%)$ \\
Trauma & $4(17 \%)$ & $5(19 \%)$ \\
Unknown & $1(4 \%)$ & $1(4 \%)$ \\
Total & 23 & 26 \\
\hline
\end{tabular}

cause of SAH. One patient with head trauma showed CAA of moderate degree. The trauma was severe, and she had epidural and subdural haemorrhages as well as $\mathrm{SAH}$, and trauma was considered to be the major cause of the SAH. The 3 patients who had primary SAH without any obvious cause had no CAA at the bleeding sites. Thus there was no case in which primary SAH was obviously attributable to CAA.

\section{SECONDARY SAH AND CAA}

A total of 125 ICHs were identified in 101 patients among the 997 necropsy cases. Among the 101 patients with ICH, 40 presented with dementia in their clinical courses, in which 6 had been diagnosed as having DAT, 30 dementia of the vascular type, and 4 dementia of mixed (Alzheimer and vascular) type. SAH as a secondary development of ICH was found in 23 patients (ages; 65-94 years with an average of 81 years, $M / F=$ $11 / 12)$ with a total of 26 haemorrhages $(2 \cdot 3 \%$ of total series and $23 \%$ of the patients with $\mathrm{ICH})$. The location of ICH is shown in table 2. Secondary SAH most frequently developed from cerebral lobar haemorrhage, followed by cerebellar haemorrhage. There was no secondary SAH following haemorrhages of the thalamus and basal ganglia, which were the most frequent and preferential sites of hypertensive ICH.

The aetiology of $\mathrm{ICH}$ in 23 patients with secondary SAH was pathologically analysed (table 3). In $11(48 \%)$ of the 23 patients, CAA of marked or moderate degree was found, and was considered to be the cause of ICH with secondary SAH (fig 1). A total of 12 CAA-related ICH with secondary SAH found in the 11 patients were observed in the following portions: 5 in the temporal lobe, 3 in the frontal lobe, 2 in the occipital lobe, and 2 in the cerebellum.

The aetiology of haemorrhage in the 101 patients with ICH (125 haematomas in total) was investigated. CAA-related $\mathrm{ICH}$ was found in 11 patients (age; 77-90 years with an average of 82 years, $M / F=4 / 7) \quad(12$ haematomas). All 11 patients with CAArelated ICH (12 haematomas) had secondary SAH. Seven presented with dementia, in which 4 had been suffering from DAT, and 3 from dementia of mixed (vascular and Alzheimer) type. Hypertension was found in 2.

Clinical records for episodes of intracranial haemorrhages were analysed for the 7 patients having CAA-related ICH with secondary SAH, and in 6 patients with secondary SAH developing from hypertensive ICH. The patients with CAA-related ICH and secondary SAH were seen with a sudden 
Figure 2 Leptomeningeal vascular changes

associated with cerebral

amyloid angiopathy

$(C A A)$. Hyalinous, onion

skin-like changes of the

intima of a leptomeningeal

vessel (arrows) $(A-C)$.

Congo red staining $(A)$

and its observation with

polarised light (B) show

amyloid deposition in the

media of leptomeningeal

vessels (arrow heads).

Amyloid deposits (arrow

heads) show green

birefringence under

polarised light (B).

Elastica-Masson staining

(C) indicates fibrous

nature of the hyalinous

intimal changes and

degeneration of the internal

elastic lamina and media.

Focal subarachnoid

haemosiderosis around a

leptomeningeal vessel with

hyaline change (D)

(A: Congo red, $\times 170$,

$B$ : Congo red under

polarised light, $\times 170$,

C: Elastica-Masson stain,

$\times 240, D$ : Congo red, $\times 145$ )

$(B a r=100 \mu m)$
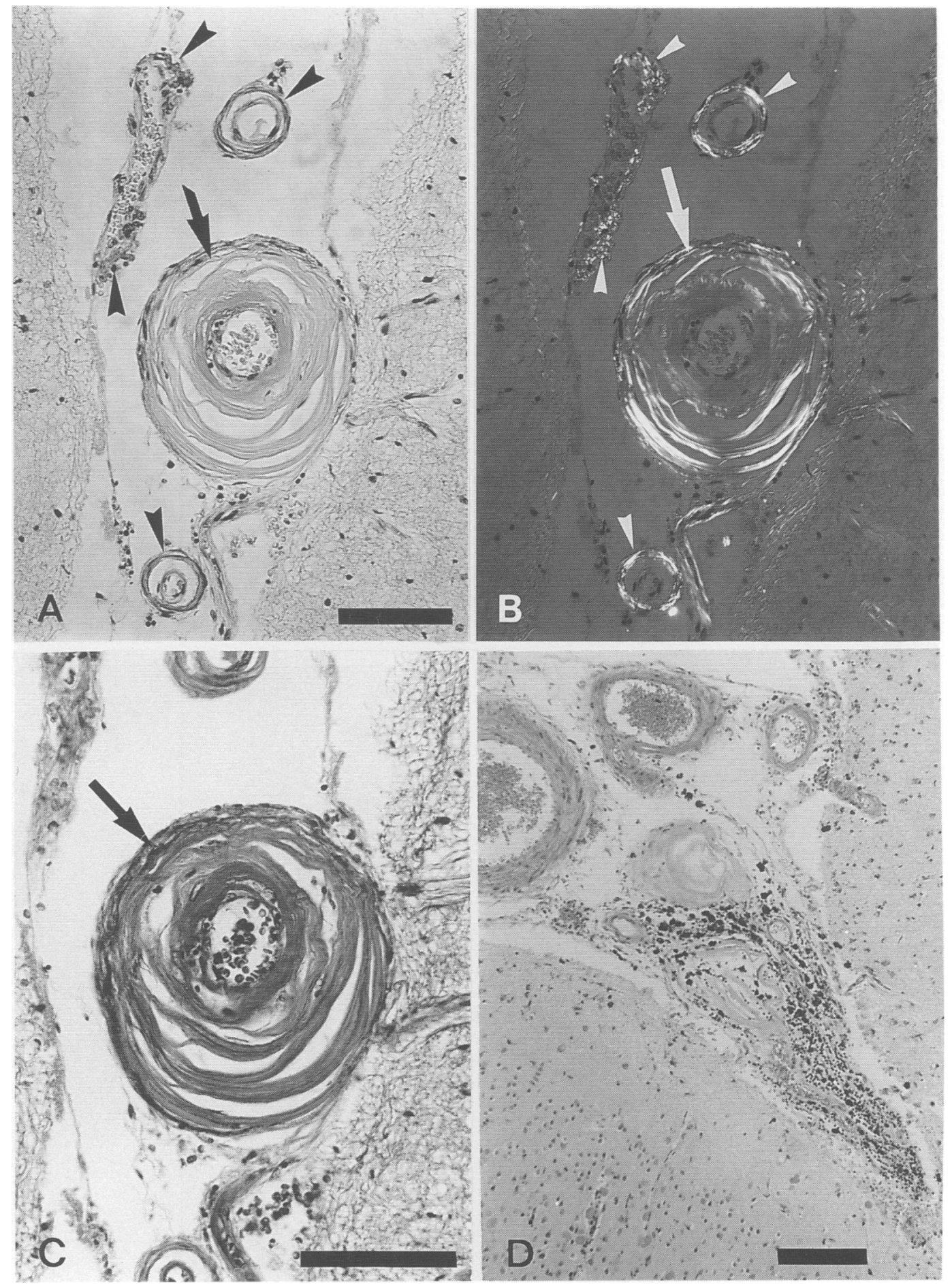

onset of disturbance of consciousness (coma or confusion) in $7 / 7(100 \%)$, focal neurological deficit in $6 / 7(86 \%)$, meningeal signs in $1 / 7(14 \%)$, and seizure in $1 / 7(14 \%)$. The patients suffering from hypertensive $\mathrm{ICH}$ with secondary SAH had a sudden onset of disturbance of consciousness in 5/6 (83\%), focal neurological signs in $6 / 6(100 \%)$, and meningeal signs in $1 / 6(17 \%)$. On CT scans, CAA-related ICH tended to be larger in size in comparison with hypertensive ones.

LEPTOMENINGEAL AND CORTICAL VESSELS IN CAA

In the brains from the patients with CAArelated $\mathrm{ICH}$, amyloid deposition of marked or moderate degree was found in leptomeningeal and cortical vessels. CAA was immunohistochemically positive for beta/A4 protein in all cases.

Leptomeningeal arteries in CAA brains frequently showed duplication ("double barreling") of the wall and obliterative intimal changes with or without amyloid deposition of the media (fig 2). The intimal changes included fibrous thickening, onion skin-like changes, and hyaline changes. The affected leptomeningeal vessels sometimes showed dilatations with disruptions of the internal elastic lamina and medial degeneration (fig 2). However, fibrinoid necrosis (degeneration) of the vessel wall as shown with 
Figure 3 Cortical vascular changes associated with cerebral amyloid angiopathy (CAA).

Fibrinoid necrosis and microaneurysm formation (arrow) of the cortical vessels $(A-C)$. Congo red staining $(A)$ and its observation with polarised light $(B)$ indicate that amyloid is present in cortical small arteries or arterioles (arrow heads), but not in the vascular segment with fibrinoid necrosis (arrows). Elastica-Masson staining (C) shows deposition of plasma proteins (fibrin-like materials) (arrow) in the necrotic wall with loss of elastic fibres. Old micro-haemorrhage (haemosiderosis) around a cortical vessel with fibrinoid necrosis of the wall (D). (A: Congo red, 110, B: Congo red under polarised light, 110, C: Elastica-Masson stain, 110, D: Congo red, 145) (Bar $=100 \mu \mathrm{m}$ )
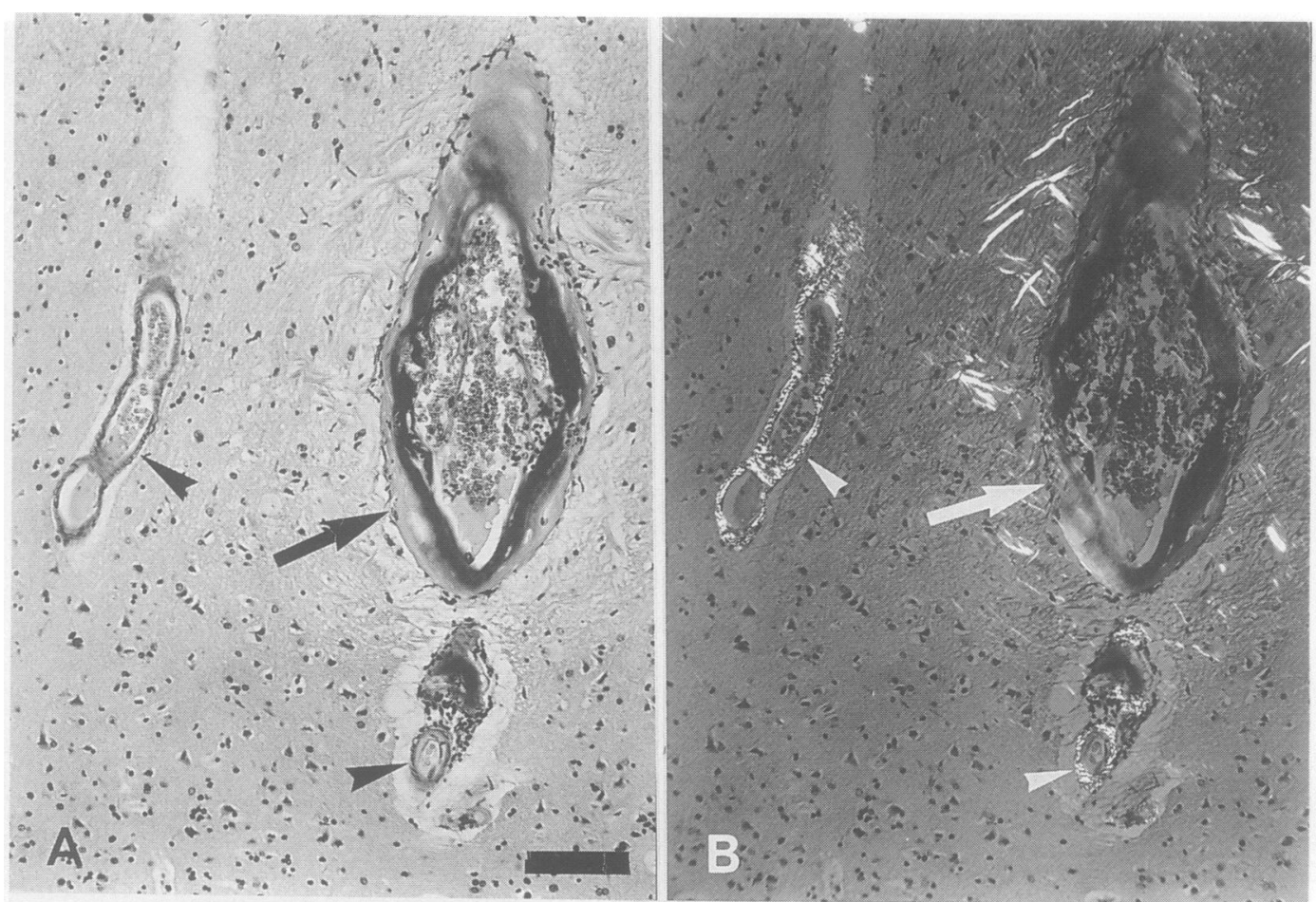

Elastica-Masson stain was rarely found in the leptomeningeal vessels. Focal haemosiderosis and lymphocytic infiltration were occasionally found around the affected leptomeningeal vessels (fig 2). The obliterative changes of leptomeningeal vessels were frequently associated with cortical infarctions.

Cortical small arteries in CAA brains frequently presented with ectatic changes or microaneurysmal dilatations and fibrinoid necrosis of vessel walls (fig 3). The cortical vessels with necrotic walls frequently accompanied perivascular fresh microhaemorrhage or haemosiderosis with gliosis, indicating that such vascular changes could be the pathology underlying CAA-related ICH (fig 3).

\section{Discussion}

In a previous study of a necropsy series from the same geriatric hospital, a CAA was identified in $57 \%$ of the total necropsy cases. ${ }^{8}$

Our results indicate that CAA rarely causes primary SAH despite the high incidence of CAA in the elderly patients. In our investigations there was no patient in which primary SAH was clearly attributed to CAA. In three patients with SAH of unknown causes, no CAA was identified. A generalised bleeding tendency was the most common cause of primary SAH in the elderly. In one patient with DIC and one patient with head trauma, CAA of moderate degree was found with primary SAH. In these cases DIC or trauma was 
considered to be the major cause of the primary SAH, although it is possible that CAA might in part contribute to the development of the SAH. Only one case with CAA and primary SAH has been described, in which the aetiological relationship between CAA and $\mathrm{SAH}$ was unknown. ${ }^{25}$

We investigated the significance of CAA as a cause of secondary SAH in the elderly. Our results demonstrated that CAA was the leading cause of secondary SAH in old age (table 3), and CAA-related ICH, located in the cerebral lobes or cerebellum, was commonly associated with secondary SAH. ICH in deep grey matter, most of which was hypertensive, did not cause secondary SAH. Some hypertensive cerebral and cerebellar haemorrhages were accompanied by secondary SAH. There have been reports suggesting that secondary SAH was frequent in CAA-related ICH, ${ }^{914-161819}$ where secondary SAH is almost constantly found on pathological examination; and clinical symptoms and signs of the meningeal involvements occur in 14-80\%.9 14-161819 Clinical manifestations of CAA-related haemorrhage are characterised by a sudden onset of disturbance of consciousness, often coma, with or without meningeal signs and focal neurological deficit.

CAA-associated vascular charges ${ }^{22}$ have been reported with cerebrovascular lesions; ;91422 23 fibrinoid necrosis (degeneration) and microaneurysm formation of cortical vessels, which are similar to changes found in hypertensive patients, would be involved in the development of CAA-related ICH. Our observations that CAA was commonly associated with ICH of cerebral lobes or cerebellum, but not with primary SAH would be explained by the differences between leptomeningeal and cortical vascular changes in the CAA brains. The leptomeningeal vessels showed hyaline change of the vascular walls, but rarely accompanied by fibrinoid necrosis. In contrast, the cortical vessels frequently showed fibrinoid necrosis of the aneurysmally dilated vascular walls. Scarcity of fibrinoid necrosis in the subarachnoid leptomeningeal vessels may be the reason for the rarity of primary SAH in CAA. However, occasional haemosiderosis microscopically found around the meningeal vessels suggests a possible CAA-related microhaemorrhage in the subarachnoid space. The finding that CAA-related ICH was commonly associated with secondary SAH would be explained by the superficial location of CAArelated vascular pathology with fibrinoid necrosis and microaneurysm formations of the cortical arteries. It requires further investigation to elucidate pathogenesis of such CAA-associated vasculopathies and to establish significance of the vasculopathies for CAA-related CVD.
In conclusion, our studies have indicated that primary SAH in the elderly is rarely related to CAA. In contrast, CAA is the most frequent cause of $\mathrm{ICH}$ with secondary $\mathrm{SAH}$ in the elderly. The fact that CAA is commonly associated with $\mathrm{ICH}$, but not with primary SAH could be related to the differences in CAA-associated vasculopathies in leptomeningeal and cortical vessels.

1 Toole JF. Vascular diseases. Etiology and pathogenesis. In: Rowland LP, ed. Meritt's textbook of neurology. 8th ed. Philadelphia: Lea and Febiger 1989:181-191.

2 Toole JF, Robinson MK, Mercuri M. Primary subarachnoid hemorrhage. In: Vinken PJ, Bruyn GW, Klawan $\mathrm{H}$, eds. Handbook of clinical neurology, Vol 55 . Vascular diseases, Pt III. Amsterdam: Elsevier 1989: 1-39.

3 Morimatsu M, Hirai S, Muramatsu A, Yoshikawa M. Senile degenerative brain lesions and dementia. $\mathcal{F} \mathrm{Am}$ Geriatr Soc 1975;23:390-406.

4 Mandybur TI. The incidence of cerebral amyloid angiopathy in Alzheimer's disease. Neurology 1975;25: 120-126.

5 Tomonaga $M$. Cerebral amyloid angiopathy in the elderly. f Am Geriatr Soc 1981;29:151-157.

6 Vinters HV, Gilbert JJ. Cerebral amyloid angiopathy: incidence and complications in the aging brain. II. The distribution of amyloid vascular changes. Stroke 1983;14 924-8.

7 Esiri MM, Wilcock GK. Cerebral amyloid angiopathy in dementia and old age. $\mathcal{F}$ Neurol Neurosurg Psychiatry 1986;49:1221-6.

8 Yamada $M$, Tsukagoshi $H$, Otomo E, Hayakawa $M$. Cerebral amyloid angiopathy in the aged. $f$ Neurol 1987;234:371-6.

9 Vinters HV. Cerebral amyloid angiopathy. A critical review. Stroke 1987;18:311-24.

10 Masuda J, Tanaka K, Ueda K, Omae T. Autopsy study of incidence and distribution of cerebral amyloid angiopathy in Hisayama, Japan. Stroke 1988;19:205-210.

11 Torack RM. Congophilic angiopathy complicated by surgery and massive hemorrhage. A light and electron microscopic study. Am $\mathcal{F}$ Pathol 1975;81:349-66.

12 Jellinger $\mathrm{K}$. Cerebrovascular amyloidosis with cerebral hemorrhage. $\mathcal{F}$ Neurol 1977;214:195-206.

13 Mandybur TI, Stephen RD, Bates MB. Fatal massive intracerebral hemorrhage complicating cerebral amyloid angiopathy. Arch Neurol 1978;35:246-8.

14 Okazaki H, Reagan TJ, Campbell PJ. Clinicopathologic studies of primary cerebral amyloid angiopathy. Mayo Studies of primary cerebral $1979 ; 54: 22-31$.

15 Tyler KL, Poletti CE, Heros RC. Cerebral amyloid angiopathy with multiple intracerebral hemorrhages. Case report. F Neurosurg 1982;57:286-9.

16 Gilbert JJ, Vinters HV. Cerebral amyloid angiopathy: incidence and complications in the aging brain. I. Cerebral hemorrhage Stroke 1983;14:915-23.

17 Finelli PF, Kessimian N, Bernstein PW. Cerebral amyloid angiopathy manifesting as recurrent intracerebral hemorrhage. Arch Neurol 1984;41:330-3.

18 Ishii N, Nishihara Y, Horie A. Amyloid angiopathy and lobar cerebral haemorrhage. $\mathcal{f}$ Neurol Neurosurg Psychiatry 1984;47:1203-10.

19 Kalyan-Raman UP, Kalyan-Raman K. Cerebral amyloid angiopathy causing intracranial hemorrhage. Ann Neurol 1984;16:321-9.

20 Cosgrove GR, Leblanc R, Meagher-Villemure K, Ethier R: Cerebral amyloid angiopathy. Neurology 1985;35: 625-31.

21. Gray F, Dubas F, Roullet E, Escourolle R. Leukoencephalopathy in diffuse hemorrhagic cerebral amyloid angiopathy. Ann Neurol 1985;18:54-9.

22 Mandybur TI. Cerebral amyloid angiopathy: the vascular pathology and complications. $\mathcal{F}$ Neuropathol Exp Neurol 1986;45:79-90.

23 Vonsattel JPG, Myers RH, Hedley-Whyte ET, et al. Cerebral amyloid angiopathy without and with cerebral hemorrhages: a comparative histological study. Ann Neurol 1991;30:637-49.

24 Yamada $M$, Tsukagoshi $H$, Otomo E, Hayakawa $M$ Systemic amyloid deposition in old age and dementia of Alzheimer type: the relationship of brain amyloid to Alzheimer type: the relationship of brain amyloid to
other amyloid. Acta Neuropathol (Berl) 1988;77:136-41.

25 Ohshima T, Endo T, Nukui H, et al. Cerebral amyloid angiopathy as a cause of subarachnoid hemorrhage. Stroke 1990;21:480-3. 